\title{
Active Omni-directional Piezoelectric Energy Harvesting System for Wireless Monitoring on Electrical Traction Shearer
}

\author{
Xuhui Zhang ${ }^{1,2}$ \\ ${ }^{1}$ Department of Mechanical Engineering, Xi'an University of Science and \\ Technology, No.58 Yatan Road, Xi'an 710054, China \\ ${ }^{2} X i$ 'an Coal Mining Machinery Co., Ltd., Xi'an 710032, China \\ zhangxh@xust.edu.cn
}

\begin{abstract}
For communication and energy supply problems caused by sensors wing in condition monitoring, a novel wireless monitoring system based on Piezolectric Energy Harvesting (PEH) and Wireless Sensor Network (WSN) were proposed to realize self-powered and intelligent detection of electrical traction shearer. With the maximum power accumulated from kinetic energy, the working condition monitoring sensors and wireless communication module were powered. By analyzing the function of modules, this paper put forward an selfpowered WSN monitoring system scheme, introduced the developing of the monitoring system which include the Omni-directional energy colleetion device structure and optimization its structure and parameter, low power energ. harvesting aircuit design, modeling of energy collection efficiency, WSN nodes design and its energ. consumption analysis. This research helps to solve the difficult in sensorcwing, communication, power supply and contribute to improve the level of condition monitoring for electrical traction shearer.
\end{abstract}

Keywords: Vibration energy harvesting, WSN, Condition Monitoring, Shearer

\section{Introduction}

Mining machinery condition monitoring is the foundation of scientific evaluation for health status and maintenarce decision of equipments. The current condition monitoring systems on coalmining nachinery are focused on electrical, hydraulic and shearer posture, such as position of shearer-in working face, pitch angle and inclination, radial height and so on. Those monitoriprg are lack of mechanical default detection. The maintenances of mining equipment or mechanical parts still remain backward mode as regular maintenance and overhaul repairing process.

Since lack of the monitoring data from mechanical parts, especially in machine driven system, researchers would be difficult to implement a scientific assessment of the health status for equipment and maintenance decisions, reducing the capacity utilization of the device, Many hidden faults are difficult to detect, resulting in frequent failures of mining machnery and become the impact of production safety in coal mine. Through information retrieval and market research, the challenges were explored on key machinery parts of online monitoring to coal mine equipments. Main difficulties are sensor wiring, communications, and energy supply.

Wireless sensor network encountered power supply problem in location tracking, environmental monitoring and other aspects in coal mine. Energy harvesting system has made many breakthroughs in recent years because development of the lower power consumption electronic devices such as sensor, MCU and RF, as well as lifting in micro energy harvesting 
power management IC technologies, This makes it possible for self-powered wireless communication systems[1,2].

Energy harvesting is a process by which energy is derived from external sources (e.g. solar power, thermal energy, wind energy, salinity gradients, and kinetic energy), captured, and stored for small, wireless autonomous devices, like those used in wearable electronics and wireless sensor networks[3,4]. Mechanical energy can be found almost anywhere that wireless sensor networks (WSN) may potentially be deployed, which makes converting mechanical energy from ambient vibration into electrical energy an attractive approach for powering wireless sensors[5]. However, different from other industries, available energy harvest mode is very limited underground in the coal mine [6]. Solar power or thermal energy are hard to use for almost no light and temperature gradients too far from the position of the nodes in the coal mine. Considering frequent vibration during operation of the mining machinery, reasonable energy conversion structures were developed as supplemental power for the rechargeable lithium battery or super capacitor $[7,8]$.

Therefore, combined the energy harvesting, WSN and modern detection technology, a novel wireless monitoring system based on active PEH was developed With the maximum power accumulated from kinetic energy, the working condition monitoring sensors and wireless communication module were powered to achieve the sensor/self-powered and intelligent detection. And the research can help to solve the wiring communication, energy supply of the sensors in condition monitoring of coal mining equipment. This paper analyzes the development of PEH-based wireless condition monitoring firstly. In Section 2, the scheme of wireless monitoring network for Shearer is reported. In Section 3, the vibration sensor node based PEH and WSN plan js explained. In Section 4, structural analysis and design of the active Omni-directional PEH arescribed in detail. The circuit of the PEH system based CC430 MCUand LTC3588 1 chip is introduced in Section 5. In Section 6, the experimental results are showrand discussed.

\section{Wireless Condition Monitoring System for Shearer}

The wired power connections are unrealistic in some applications, such as remote monitoring system of railway based on wireless sensors [9], remote measurements [10], GPS tracking and location system for vare wild animal [3], vibration monitoring for buildings structure with sensors embedded in the internal of structure, video monitoring and locating system used in military field and so on. For the coal mining machinery, the cable power supply mode means complex wiring channel and explosion-proof structure. Some key machinery parts carmot monitor because the sensors are difficult to install. In a representative rotating machinety monitoring and fault diagnosis system used by domestic manufacturers, the number of detection sensors mounted on a shaft may about a dozen. If the monitoring system aimed at a comprehensive detection for mining machinery, measuring point, quantity of detection and sensors will be more.

The aspects of energy harvesting system were researched by scholars all over the world. Energy can also be harvested to power small autonomous sensors such as those developed using MEMS technology. Scavenging energy from ambient vibrations, wind, heat or light could enable smart sensors to be functional indefinitely. Several academic and commercial groups have been involved in the analysis and development of vibration-powered energy harvesting technology, including Control, Power Group, Optical and Semiconductor Devices Group at Imperial College London, and IMEC, MIT Boston, Georgia Tech, UC Berkeley, Southampton University, Columbia University. The National Science Foundation also supports an Industry/University Cooperative Research Center led by Virginia Tech and The University of Texas at Dallas called the Center for Energy Harvesting Materials and Systems. 
Typical power densities available from energy harvesting devices are highly dependent upon the specific application (affecting the generator's size) and the design itself of the harvesting generator. In general, for motion powered devices, typical values are hundreds of $\mu \mathrm{W} / \mathrm{cm}^{3}$ for generators powered from machinery [11].

Integrated the WSN and active PEH technologies, this paper presented a vibration-based energy harvesting wireless monitoring system for coal mining machinery to meet the demand of condition monitoring on mine equipment. The overall design of wireless monitoring network for underground mining machinery is shown in Figure 1.

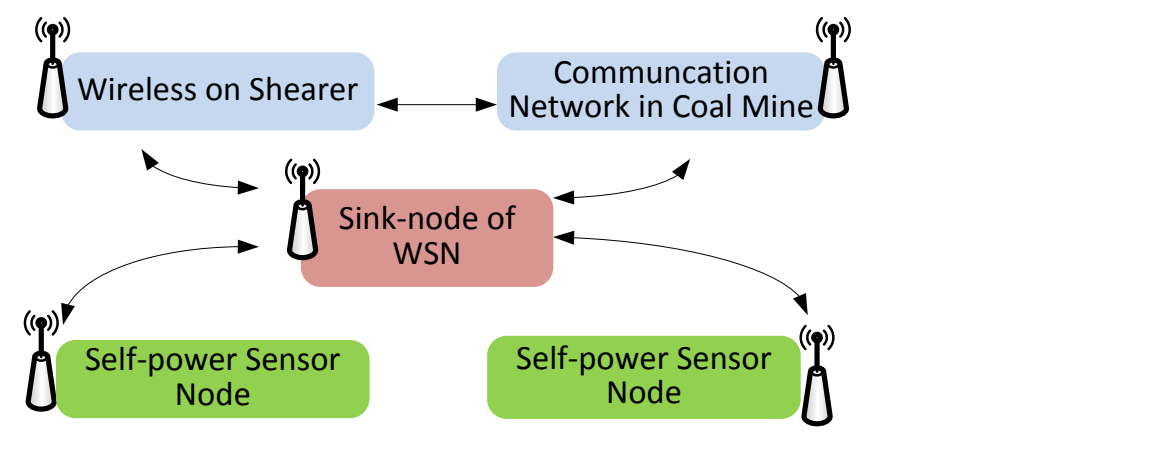

Figure 1. Schematic Diagram of Wireless Monitoring Network for Shearer

The system consists of self-powered wreless senser nodes, sink-nodes of WSN and communication module on Shearer. The Wineless sensor nodes manage the PEH devices and process vibration signal from key machinery parts vibration of shearer during working. In accordance with the signal features of vibration the wireless sensor node decides whether to upload data to the sink-node by the communication module on Shearer. The sink-nodes can also save the signal features of shearer, and periodically read the monitoring data to process fault trend forecasting offline analysis. This process is just as "black box" function on airline. The sink-nodes conmected with shearer and underground communication network to upload the monitoring data, and eventually chieve the remote monitoring and fault diagnosis process.

\section{Design Scheme of Vibration Sensor node based PEH and WSN}

The self-powered wireress vibration sensor node designing scheme is shown in Figure 2. The sensor node consists of four parts: Omni-directional PEH module, signal conditioning module, MCU, unit with data acquisition function and wireless communication module.

A wireless vibration sensor node works as follows: the piezoelectric energy harvesting system adopted a piezoelectric transducer to convert vibration energy of environment or mining machine body into electrical energy. With the buck Chopper circuit and power management chip, the energy storage module charged electrical energy on L-ion battery or super capacitor. The energy storage module provides power for data collection, wireless communication of system. The sensor collects vibration signal (or temperature, pressure and other condition monitoring sensors) from key parts of shearer. After processed by conditioning circuit, the signals were sent to MCU. The MCU performed A/D, data storage, and signal per-process and wireless communication. The wireless communication module based on CC430 MCU received the data from sink-nodes and communicated with the underground Industrial Ethernet. 


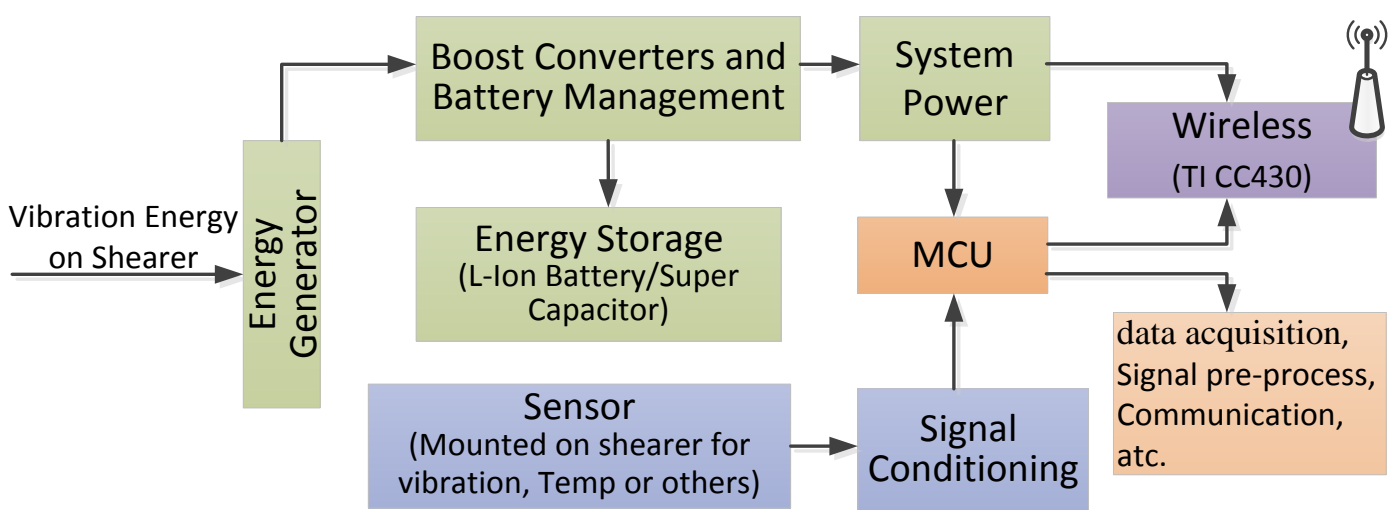

Figure 2. Schematic Diagram of Wireless Vibration Sensor Node with PEH

Analyzed the process of vibration energy collection, all sort of factors were considered. The factors include the size of the local oscillator and the vibration energy, selection of piezoelectric materials and its energy conversion efficiency, energy storage and signal conditioning circuitry, the balance between power supply and power consumption, wireless communication and system size[12, 13].

\section{Structural Analysis and Design of the Omni-directional Active PEH}

\subsection{Comparison and selection of Piézoelectric Material}

Some scholars have researched piezoelectric materials and the structural of energy converter. USA Pennsylvania State University Hofmarin.HF, Yen Massachusetts Institute of Technology, BC, University of Michigan, and Jonathan Granstrom carried out fruitful work. Vibration energy harvesting products have applied in industry. The well-known product is Harvestor-III Power Niodule developed by U.S. Advanced Cerametrics, Inc. (ACI). Its appearance, parameter and charging performance are shown in Figure 3.

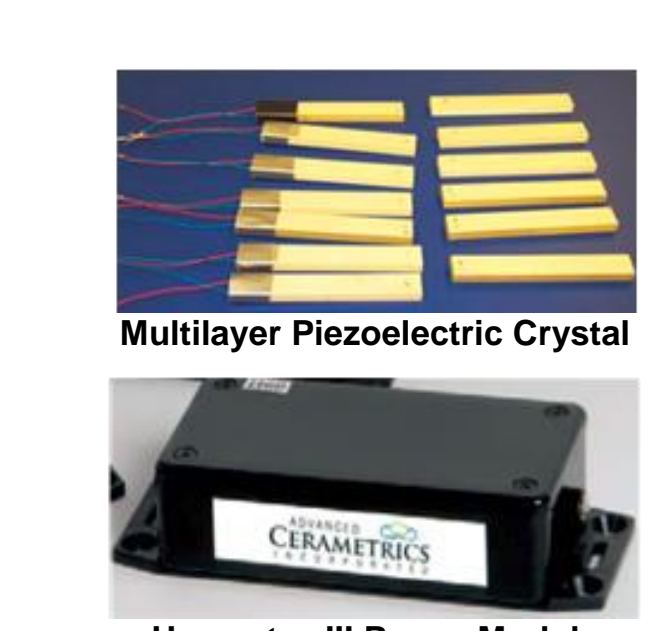

Harvestor-III Power Module

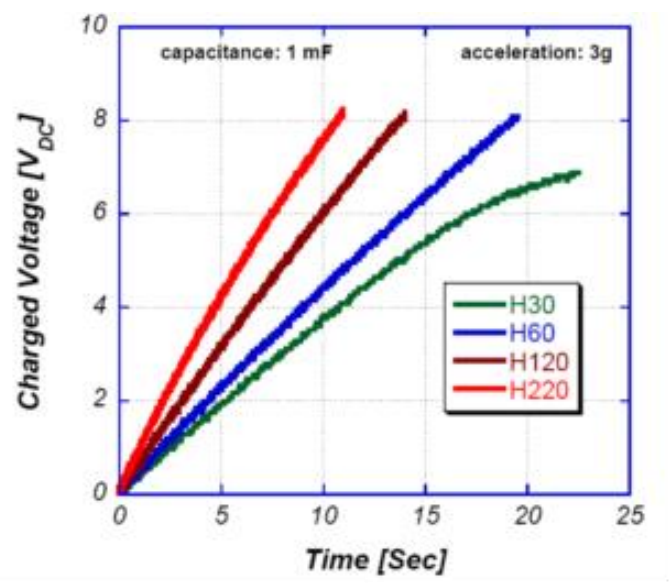

Charging Performance

Figure 3. Harvestor-III Power Module from Advanced Cerametrics, Inc. 
Two piezoelectric materials performances were compared synthetically, piezoelectric ceramics and Polyvinylidene Fluoride (PVDF). The electromechanical coupling coefficient of piezoelectric ceramic can be up to 0.313-0.694 (Plumbum zirconate titanate ceramic, PZT series), and apt to process and choose polarised direction. The PZT ceramics have widely used in energy transfer, such as the guided missile igniter, lighters ignition, etc. The PZT-5 is suitable for vibrant energy transfer component for its high resistivity, high-tension electricity emergency constant and high electromechanical coupling coefficient. The PVDF can working without power since it produces electric signal between the surfaces of upper and lower electrodes when the piezoelectric film being drawn or crooked. PVDF is very sensitive to dynamic stresses, the typical sensitivity values up to $10-15 \mathrm{mV}$ per micro train of a $28 \mathrm{um}$ PVDF. So PVDF were used in medical field extensively as the transducer to measure the slight signal. Through experimental analysis, PZT was chosen as energy harvesting component for the PEH device design.

\subsection{Modeling of PEH Device under Dynamic Loading}

The working vibration energy collector can be regarded as a SDOF/single degree of freedom) simple vibration movement caused by excitation force of forced vibration. Inertialbased kinetic energy harvesters are modeled as second-order, spring-mass systems. The generic model of kinetic energy harvesters was first developed by Williams and Yates [4]. Figure 4 shows a generic model of such a generator. The model mainly includes the generator housing, seismic mass, $\mathrm{m}$, spring with spring constant of Kand damping with damping coefficient $\mathrm{c}$.

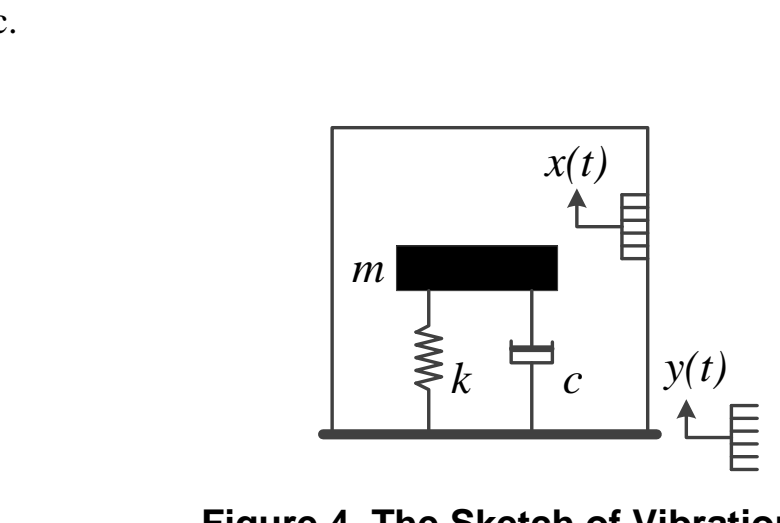

Figure 4. The Sketch of Vibration Model

When the generator vibrates, the mass moves out of phase with the generator housing. There is a relative movement between the mass and the housing. This displacement is sinusoidal in amplitude and can drive a suitable transducer to generate electrical energy. Therefore if we can calculate the power generated by damping while vibration occurs, the affecting actors of the PEH device can be analyze.

The generator housing movement is simple harmonic motion sinusoidal. For a sinusoidal excitation, the displacement of the generator housing $\mathrm{x}(\mathrm{t})$ can be written as $x(t)=x_{0} \sin \omega t$, where $x_{0}$ is the amplitude of vibration and $\omega$ is the angular frequency of vibration. $y(t)$ is the relative motion of mass with respect to the housing [14].

For the analysis, it is assumed that the mass of the vibration source is much greater than the mass of seismic mass in the generator and the vibration source is unaffected by the movement of generator. According to Newton's second law, the differential equation of the movement 
with respect to the generator housing from the dynamic forces on the mass can be derived as follows:

$$
m[\ddot{y}(t)+\ddot{x}(t)]=-k\left[y(t)+\delta_{s}\right]-c \dot{y}(t)+m g
$$

Where $\delta_{s}$ is spring Static elongation under gravity, $\delta_{s}=m g$, according to Hooke's Law. $\delta_{s}$ substitutes into equation 1 .

$$
m \ddot{y}(t)+c \dot{y}(t)+k y(t)=-m \ddot{x}(t)
$$

This can get steady-state solutions of damping relative displacement after $x(t)$ substitutes into equation 2 .

$$
y(t)=y_{0} \sin (\omega t+\theta)=\frac{\omega^{2}}{\sqrt{\left(\frac{k}{m}-\omega^{2}\right)^{2}+\left(\frac{c \omega}{m}\right)^{2}}} x_{0} \sin (\omega t+\theta)
$$

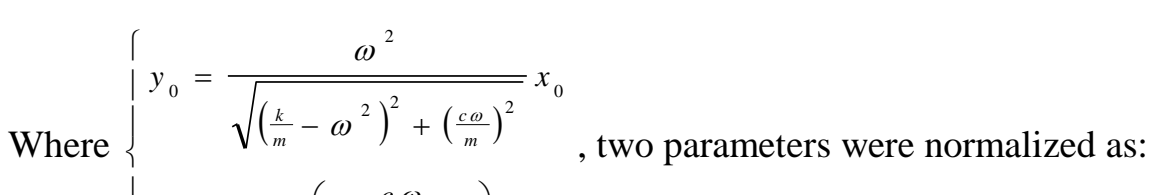

$$
\begin{aligned}
& \mid \theta=\arctan \left(\frac{c \omega}{m \omega^{2}-k}\right) \\
& \left\{\begin{array}{l}
\frac{y_{0}}{x_{0}}=T_{r}=\frac{\lambda^{2}}{\sqrt{\left(1-\lambda^{2}\right)^{2}}} \\
\theta=\arctan \left(\frac{2 \zeta \lambda}{\left.z^{2}-1\right)}\right)
\end{array}\right.
\end{aligned}
$$

Where $T_{r}$ is the transfer rate 0 relative movement, $\lambda$ is frequency ratio, $\lambda=\omega / \omega_{n}$, $\omega_{n}=\sqrt{k} / m, \omega_{n}$ is the system natural frequency, The $\omega_{n}$ only related to the inertia components and elastic element. $\zeta$ is damping ratio, $\zeta=\frac{c}{2 m \omega_{n}}$.

The damping components play a role as obstacle and consumer. The average of energy consumption as

$$
2 P_{a v}=\frac{1}{T} \int_{0}^{T} c \dot{y}(t) * \dot{y}(t) d t=\frac{m \zeta \cdot x_{0}^{2}\left(\frac{\omega}{\omega_{n}}\right)^{3} \omega^{3}}{\left[1-\left(\frac{\omega}{\omega_{n}}\right)^{2}\right]^{2}+\left[2 \zeta\left(\frac{\omega}{\omega_{n}}\right)\right]^{2}}
$$

When $\omega=\omega_{n}$, the average power consumption of damping is maximum, the theoretical value is

$$
P_{a v_{-} \max }=\frac{m x_{0}^{2} \omega_{n}^{3}}{4 \zeta}
$$


The Eq. 2 shows the proportional relative between average power of energy harvesting and $m, \omega_{n}$ and $x_{0}$. From above analysis, we can get the basic principles to design a vibration energy harvesting device as follows: (1) Vibration frequency of device equal to natural frequency. (2) The bigger quality of seismic mass. (3) The larger amplitude of seismic mass. (4) Minimize the ratio of the damping system.

\subsection{The Omni-directional Vibration Energy Collecting Device}

In kinetic energy harvesting, a piezoelectric transduction mechanism is used to extract electrical energy from motion. The generator also requires a mechanical system to couple environmental displacements to the transduction mechanism. The mechanical system has to be designed to maximize the coupling between the mechanical energy source and the transduction mechanism. Most vibration-based micro-generators are single degree of freelom second-order spring-mass system consisting of an inertial frame that transmits the vibration to a suspended inertial mass to produce a relative displacement or cause mechanical strain. Using the relative displacement or strain, the electrical energy can be generated on transduction mechanism.

Considering above analysis and vibration characteristics of mining process, this paper proposed a novel PEH device, a kind of harvester using spring-mass structure to achieve the multi-directional vibration energy collecting. The symmetric structure make random excitation on generator housing convert maximum, and colleccenergy more effectively.

A variety of PEH devices were comparaive analysis [14 15], such as internal-external cylindrical structure, cube-mass-vertex elastomer structare based on paste piezoelectric film, cube-mass-symmetric elastomer structure using PVDF, cube-mass-symmetric elastomer structure which mounted piezoelectric ceramic and so on. Although the cube-mass-symmetric elastomer structures are the research focus for its symmetric structure design to harvest multidirection vibration, these vibration model belongs to the fixed boundary cantilever. This structure which mounted the PVDF or the PZF material on cantilever is not suitable for nonvertical exciting vibration

The Omni-directional PEH structure is proposed in this paper. Its structure and working principle describe as follow: The generator housing is cubic structure, a seismic mass among the center of gubic shell. The metal mass connected with six PZT components mounted center of the six surface in cubic stricture by six spring and guide bar. In practical applications, the device can be fixed to on hearer vibration detecting position or other vibration units to harvest energy for sensor and MCU part. Since the elastic element, the vibration direction of exciting force may be any direction. This structure is one way to solve the mount question which need vertical with the vibrating direction. This 3D prototype of this PEH device is shown in Figure 5.

After mathematical modeling and analysis of the Omni-directional PEH device, main parameters of device were initially determined, include bracket side of cube, the side length and mass of cubic seismic mass, spring stiffness and other component size. In order to enhance the conversion efficiency, vibration performance researches were carried out with the Finite Element Analysis (FEA) software and optimized some parameters of the device [16]. For length of the paper, the optimize works based on FEA are not describe in detail. 


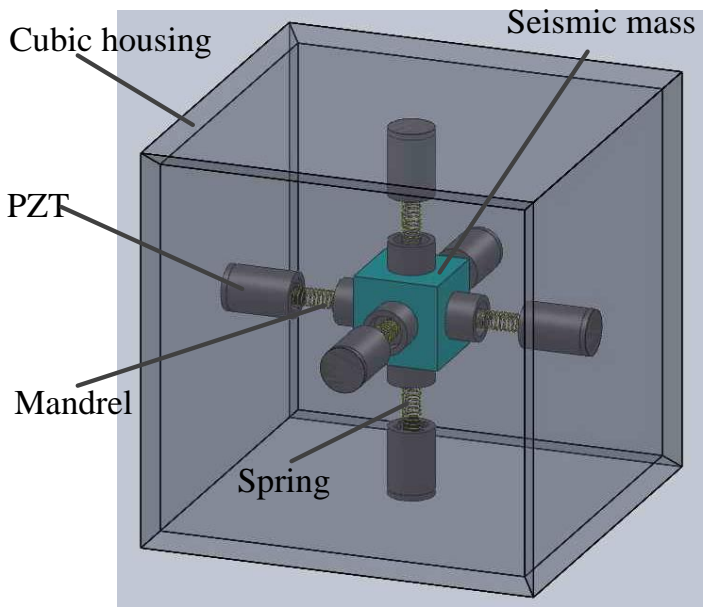

Figure 5. 3D Prototype of Omni-directional PEH Device

\section{Circuit Design of Multistage Piezoelectric Vibration Energy Harvestor}

\subsection{Output Power Modeling of Kinetic Energy Harvesters}

As mentioned above, damping in kinetic energy harvesters consists of mechanically induced damping and electrically induced damp ng[3][14]. The overall damping factor of the system, $\zeta_{T}$, is given by

$$
\zeta_{T}=\frac{b}{2 m \omega_{r}}=\frac{b_{m}+b_{e}}{2 m \omega_{r}}=\zeta_{r}
$$

The maximum dissipated power in equation (6) is given as:

$$
P_{a v_{-} \max }=\frac{\overrightarrow{m x} v^{2} \omega_{m}^{3}}{4\left(\zeta_{m}+\zeta_{e}\right)}
$$

Total quality factor (Q-factor) is a function of damping factor. It is given by $Q_{T}=1 / 2 \zeta_{T}$.

The relation between total quality factor and the electrical and mechanical damping is given by

$$
\frac{1}{Q}=\frac{1}{Q_{O C}}+\frac{1}{Q_{e}}
$$

where $Q_{o c}=1 / 2 \zeta_{m}$ is the open circuit Q-factor which reflects the mechanical damping. $Q_{e}$, which equals $1 / 2 \zeta_{e}$, reflects performance of the transduction mechanism. It cannot be measured directly, but can be calculated using Eq. (1.9) once $Q_{T}$ and $Q_{O C}$ are measured.

The power dissipation is the sum of maximum electrical energy extracted by the transduction mechanism, $P_{e}$, and mechanical loss, $P_{m} . P_{e}$ and $P_{m}$ are as follows:

$$
P_{e}=\frac{\zeta_{e} m x_{0}^{2} \omega_{n}^{3}}{4\left(\zeta_{m}+\zeta_{e}\right)}
$$




$$
P_{m}=\frac{\zeta_{m} m x_{0}^{2} \omega_{n}^{3}}{4\left(\zeta_{m}+\zeta_{e}\right)}
$$

Maximum power conversion from mechanical domain to electrical domain occurs when $\zeta_{e}=\zeta_{m}$, i.e., damping arising from the electrical domain equals mechanical losses. Therefore, the maximum electrical power that can be extracted by the kinetic energy harvester, $P_{e}$, is given by

$$
P_{e}=\frac{P_{a v_{-} \max }}{2}=\frac{m x_{0}^{2} \omega_{n}^{3}}{16 \zeta_{m}}
$$

Since the peak acceleration of the base, $a$, is given by $a=\ddot{x}=x \omega_{n}^{2}$, Eq. (12) can be rewritten as

$$
P_{e}=\frac{m a^{2}}{16 \omega_{n} \cdot \zeta_{m}}
$$

As the open circuit Q-factor, $Q_{o c}=1 / 2 \zeta_{m}$, Eq. (13) can be written as

$$
P_{e}=\frac{m a^{2}}{8 \omega_{n}} \cdot Q_{o c}
$$

It is found via Eq. (14) that the maximum power delivered to the electrical domain is inversely proportional to the damping factor, i.e. proportional to the Q-factor. Hence, when designing a vibration-based micto-generator to achieve maximum power output, it is important to design the generator with a high Q-factor (i.e., low damping factor) and make the generator work at its resonant frequency. Since the output power is inversely proportional to the resonant frequeney of the generator for a given acceleration, it is generally preferable to operate at the lowest available fundamental frequency.

\subsection{PEH En ergy Harvesting Module Design}

Vibration energy collection subsystem design is the core of the entire system. The main function of the vibration gnergy collection device is converted environmental vibration source (Shearer) vibration energy to electrical energy [17]. And supplied power for MCU and wireless by the energy collecting circuit, include rectifier, filter, and energy storage circuit [18] [19]. LTC3588-1 chip from Linear Technology Inc. was elected as energy conversion control chip. Figure 6 shows the typical application circuit of LTC3588-1 chip. The collect energy can be stored in a capacitor, super capacitor, or battery. Capacitors are used when the application needs to provide huge energy spikes. Batteries leak less energy and are therefore used when the device needs to provide a steady flow of energy.

LTC3588-1 integrates a low-loss, full-wave bridge rectifier and a high efficiency buck converter. The chip is helpful for researcher to harvest vibration energy in equipment or environment by piezoelectric materials. Though processed low-loss, full-wave bridge rectifier and a high efficiency buck converter, the vibration energy was converted into a well regulated output for microprocessor, sensors, and wireless power consumption. The pulse load can up to $100 \mathrm{~mA}$ of continuous output current or even higher. The output voltage can be separately set according to the different needs as $1.8 \mathrm{~V}, 2.5 \mathrm{~V}, 3.3 \mathrm{~V}$ or $3.6 \mathrm{~V}$. When the output is no-load, 
the quiescent current is only 950nA. The overall efficiency is maximizing improved for above characteristics.

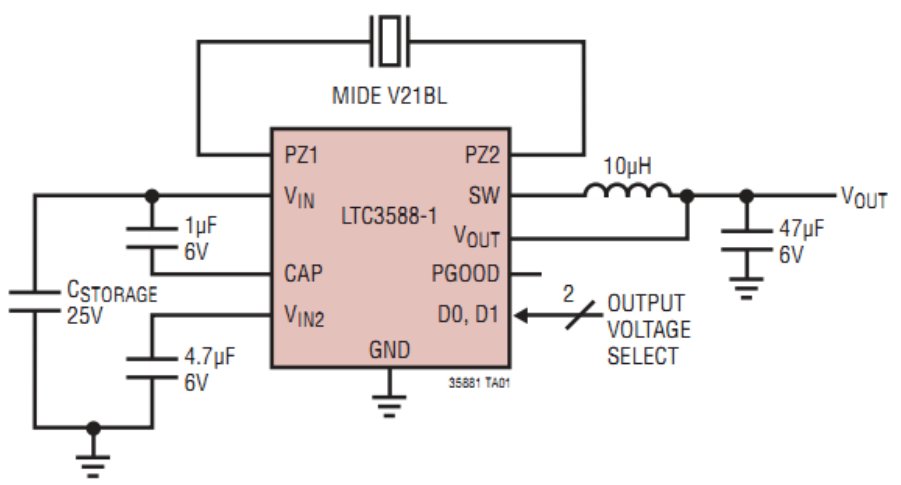

Figure 6. Wiring and Testing Circuit of LTC3588-1

\subsection{Design of Signal Acquisition and Communication Modules}

The signal acquisition and communication module mainly complete signal conditioning, A/D function and communication with external system. After signal conditioning and A/D, condition monitoring signal were sent to wireless communication module. Under shearer working, the wireless communication module sent sensors data, while received external signal. It is important to design the communication modules because its power consumption account for a large proportion of low-power systent. The CC430F6147 CC1101 system uses ultra-low power micro-controller systern, which is equipped with an integrated RF transceiver core. The powerful MSP430 RISC CPU represents the current level of technological development. A $1 \mathrm{GHz}$ RF Transceiver comibines with MSP430 CPUXV2. The MCU include a $32 \mathrm{kB}$ system programmable flash memory, a $4 \mathrm{kB}$ RAM, two 16-bit timers, 8 highperformance $\mathrm{A} / \mathrm{D}$, comparator, a universal serial communication interface (USCI), a 128bit AES security accelerator, hardware multiplier, real-time clock module with alarm capabilities , LCD driver, and up to $44 \mathrm{I} / \mathrm{O}$ pins.

\section{System Simulation Analysis and Experiment}

The prototype of Omni-directional piezoelectric energy harvesting wireless monitoring system is shown jn Figure 7. The acceleration sensor shown in FIG sensor Shearer practical applications can be a pressure measurement, a temperature sensor or other position and attitude. Converted energy of Vibration energy harvesting module is an energy supplement by charge the battery under continue working conditions. The rechargeable battery supplied power o system circuitry. The circuits can avoid power fluctuation caused by inconsistent of vibration energy harvesting on different loads.

Consider using the vibrational energy as system power, the main energy consumption includes three parts, MCU complete data acquisition and signal processing calculations, wireless communication and vibration energy harvesting module controls. The power consumption of the overall system was estimated refer to the on the data sheets of CC430, LTC3588-1 and peripheral circuits. The estimates data is shown in Table 1. 


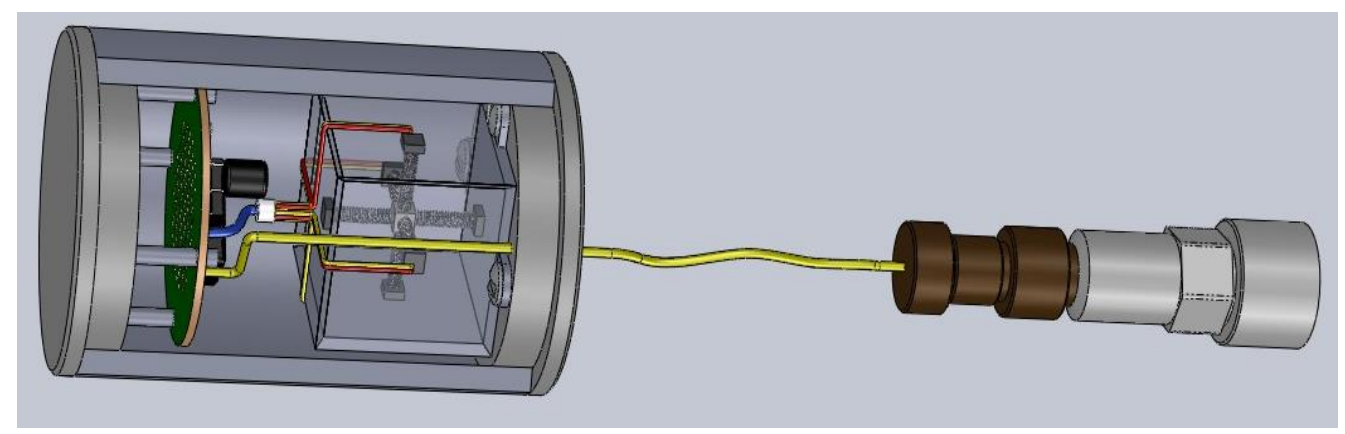

Figure 7. 3D Sketch of Self-powered Sensor

Table 1. Power Consumption of the Sensor Node based PEH and WSN

\begin{tabular}{ccc}
\hline Module & Voltage $(\mathrm{V})$ & Current $(\mu \mathrm{A})$ \\
\hline MCU(Active Mode) & 3 & 160 \\
MCU(Stand by Mode) & 3 & 2.0 \\
Wireless Communication & 3 & $15000^{*}$ \\
PEH & 3 & 250 \\
\hline *CC430 test parameters at $250 \mathrm{kbps}, 915 \mathrm{MHz}$ & &
\end{tabular}

*CC430 test parameters at 250kbps, $915 \mathrm{MHz}$.

Using the results of optimization analysis and FEA simalation, a PEH device was developing. The prototype of PEH device Is shown in Figure 8. We design an experimental table to verify the possibility of power quantity which convert from the vibrational energy. In testing, we shake the PEH device along a direction perpendicular vibration mode. The mass compressed spring and the mandrel bit on piezoelectric ceramic. To measure output voltage on piezoelectric ceramics, a Tektronix oscilloscope was used. The waveform of voltage is shown in Figure 9. In the figure, maximum output voltage is $20 \mathrm{~V}$, average voltage is $606 \mathrm{mV}$ under unidirectional vibration excitation system.

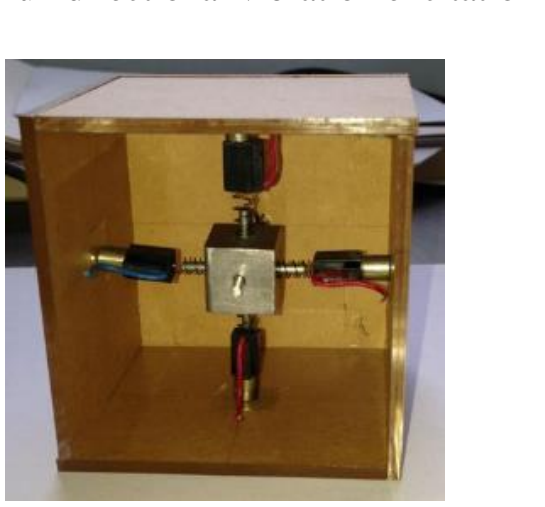

Figure 8. Prototype of PEH Device

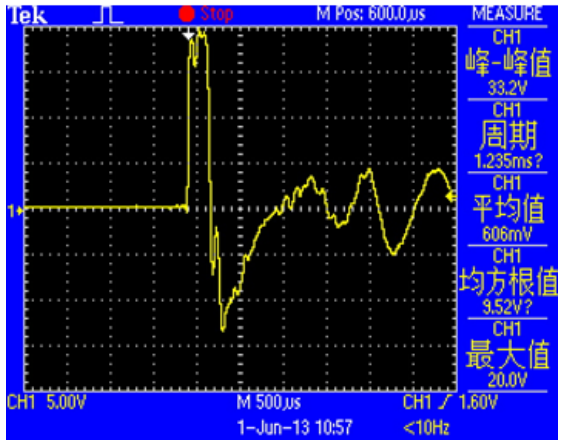

Figure 9. Output Voltage of One Direction

\section{Conclusion}

Aimed at power supply difficulties in practical application or existing equipment required to constantly replace batteries or recharged battery case in long-term use, this paper provided a vibration energy harvesting device for wireless monitoring system. Through modeling of PEH device under dynamic loading, the structure and main size of the PEH with PZT were designed. Some tests were carried out to verify the project scheme. The experiment technical 
analysis and preliminary tests showed that the energy harvesting module can meet power requirements of signal converting and processing modules, sensors, and wireless communication.

Some aspects of the design and application is not perfect in energy harvesting circuits, power conversion efficiency theory research and wireless communications. There are still many deficiencies to attain the self-powered for wireless communication and MCU operation. The further research will be built around circuit in $\mathrm{PEH}$, reduce energy consumption, precise measurement of active energy collection under mining conditions.

\section{Acknowledgements}

Part of this work is supported by the National Natural Science Foundation Project (U1361121), Natural Science Basic Research Plan in Shaanxi Province of China (2012JM8093) and Scientific Research Program Funded by Shaanxi Provincial Education Department. The author acknowledges the support from the students, Zhongfang Xan, Tengda Liu and Ran Lin for their assistance in the experiments and modeling

\section{References}

[1] C. Knight, J. Davidson and S. Behrens, "Energy Options for Wireless Sensor Nodês", Sensors, vol. 8, (2008), pp. 8037-8066.

[2] G. Yong-feng, Z. Gao-fei, W. Xiao-feng and Y. Zherg, A Miniature Composite Energy Harvesting System for Wireless Sensor Nodes", Nanotechnology and Precision Engineering, Vol. 10, no. 4, (2012), pp. 327-331.

[3] S. Priya, D. J. Inman, J.-Q. Huang and Q.-N Huang, "Energy Harvesting Technologies", Southeast University Press, Nanjin, (2011).

[4] T. J. Kaźmierski and S. Beeby, "Energy Harvesting Systems: Principles, Modeling and Applications", Springer-Verlag New York Inc., New Yok, (2011).

[5] L. Xiang-jian and C. Ren-wen, "Current situation and developing trend of piezoelectric vibration energy harvesters", Journal of Vibrationand Shock, vol.31, no 16, (2012), pp. 169-177.

[6] D. En-jie, W. Dan, J. Long-jiao and A. Lu, "Design on Enemy Collection System in Underground Mine Based on Piezoelectric Effect", Coal Science and Technology, vol. 40, no. 12, (2012), pp. 71-76.

[7] P. Harrop, "Energy Haryesting and Storage for Electronic Devices 2010-2020", ID Tech Ex Ltd., (2010).

[8] S. R. Anton and H A Sodano, "A revie of power harvesting using piezoelectric materials", Smart Mater Struct, vol. 16, no 3, (2007), pp. 2 r.

[9] A. Soma De Pasquale and F. Fraccarollo, "Piezoelectric energy harvesting for autonomous sensors network on safety-mproved railway vehicles", Proceedings of the Institution of Mechanical Engineers, Part C: Journal of Mechanical Engineering Science, vol. 226, no. 4, (2012), pp. 1107-1117.

[10] E. Sardini and M. Serpel loni. "Passive and Self-Powered Autonomous Sensors for Remote Measurements", Sensors, vol. 9, (2009), pp 943-960.

[11] Energy harvesting,http.//en.wikipedia.org/wiki/Energy_harvesting, (2013).

[12] E. Lefeuvre, G. Sebald, D. Guyomar, M. Lallart and C. Richard, "Materials, structures and power interfaces for efficient piezoelectric energy harvesting", J Electroceram, vol. 22, (2009), pp. 171-179.

[13] J. Liang and W.-H. Liao, "Impedance matching for improving piezoelectric energy harvesting systems", Proc. of SPIE, vol. 7643, (2010).

[14] C. Ren-wen, "New Ambient Energy Harvesting Technology", National Defence Industry Press, Beijing, (2011).

[15] H Zhi-wei, C. Ren-wen and L. Xiang-jian, "Optimization design of multi-direction piezoelectric vibration energy harvester", Journal of Vibration and Shock, vol. 31, no. 16, (2012), pp. 33-38.

[16] A. Sanchez, S. Blanc, S. Climent, P. Yuste and R. Ors, "SIVEH: Numerical Computing Simulation of Wireless Energy-Harvesting Sensor Nodes”, Sensors, vol. 13, (2013), pp. 11750-11771.

[17] A. Dolgov, R. Zane and Z. Popovic, "Power Management System for Online Low Power RF Energy Harvesting Optimization", IEEE Transactions on Circuits and Systems-I: Regular Papers, vol. 57, no. 7, (2010).

[18] Y.-Y. Chen, D. Vasica1, F. Costa, W.-J. Wu and C.-K. Lee, "A self-powered switching circuit for piezoelectric energy harvesting with velocity control”, European Physical Journal Applied Physics, vol. 57, no. 3, (2012), pp. 30903-30912. 
[19] G. Yi, "Research on Key Technologies of Energy Self-sufficient and Low Power Wireless Sensor Network", Doctoral Dissertation, Nankai University, Tianjin, (2009).L. Fan-qiang, M. Xiao-min and X. Xinhong, "CC430-based wireless sensor network platform and Practice", Beijing university of aeronautics and astronautics press, Beijing, (2012).W. Xian-long, "CC430 and Wireless sensor network", Metallurgical Industry Press, Beijing, (2011).

\section{Author}

Xuhui Zhang, He received his M.Sc. in Mechanical Manufacture and Automation (2002) from Xi'an University of Science and Technology and PhD in Instrument Science and Technology (2009) from Xi'an Jiaotong University. Now he is an associate professor of Mechatronics at Department of Mechanical Engineering, Xi'an University of Science and Technology. Since 2010 he is dean ôf mechatronics department. He is also working as a Postdoctoral in Xi'an Coal Mining Machinery Co., Ltd. His current research interests include different aspects of intelligent detection and control systems.

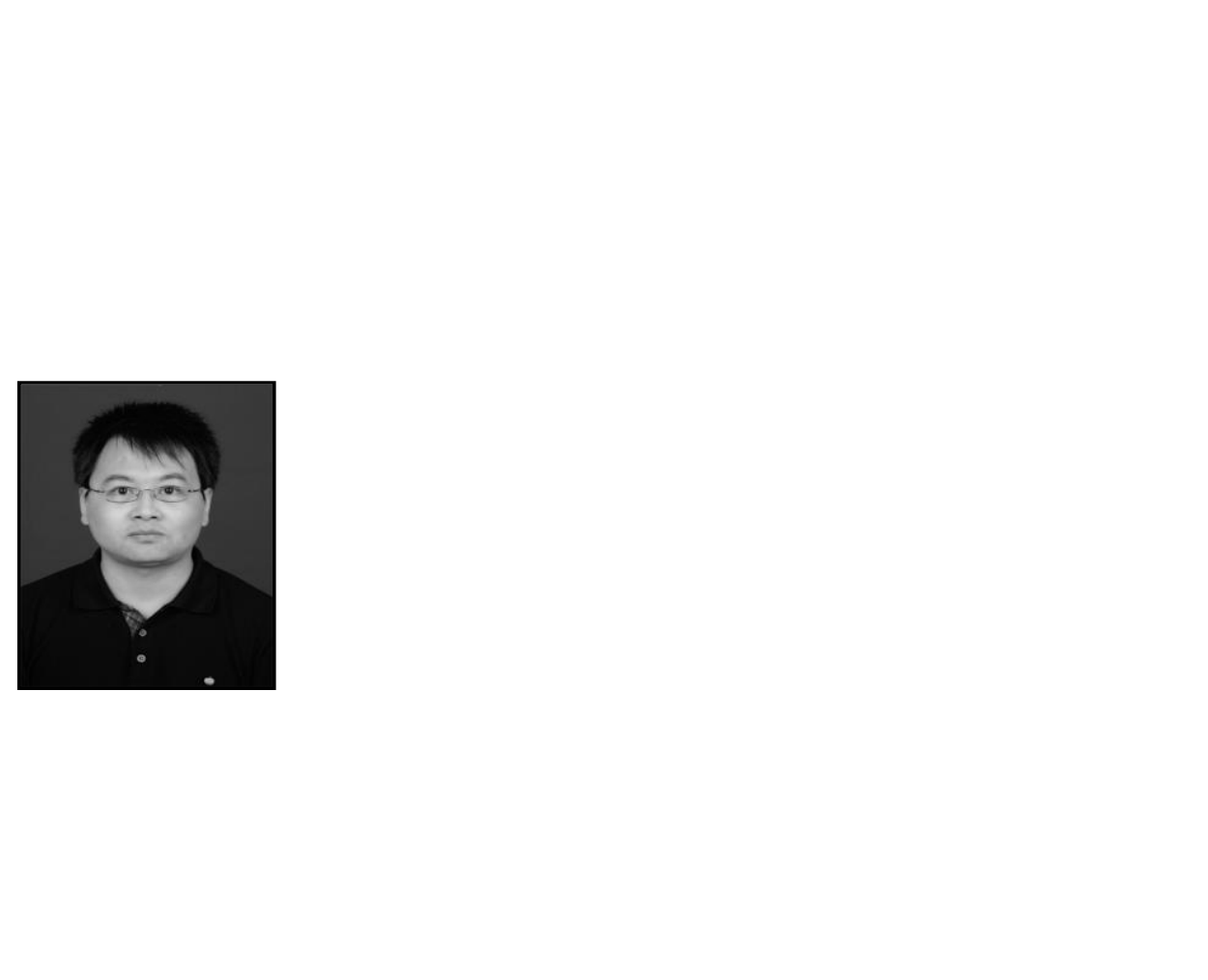


International Journal of Smart Home

Vol.8, No.3 (2014)

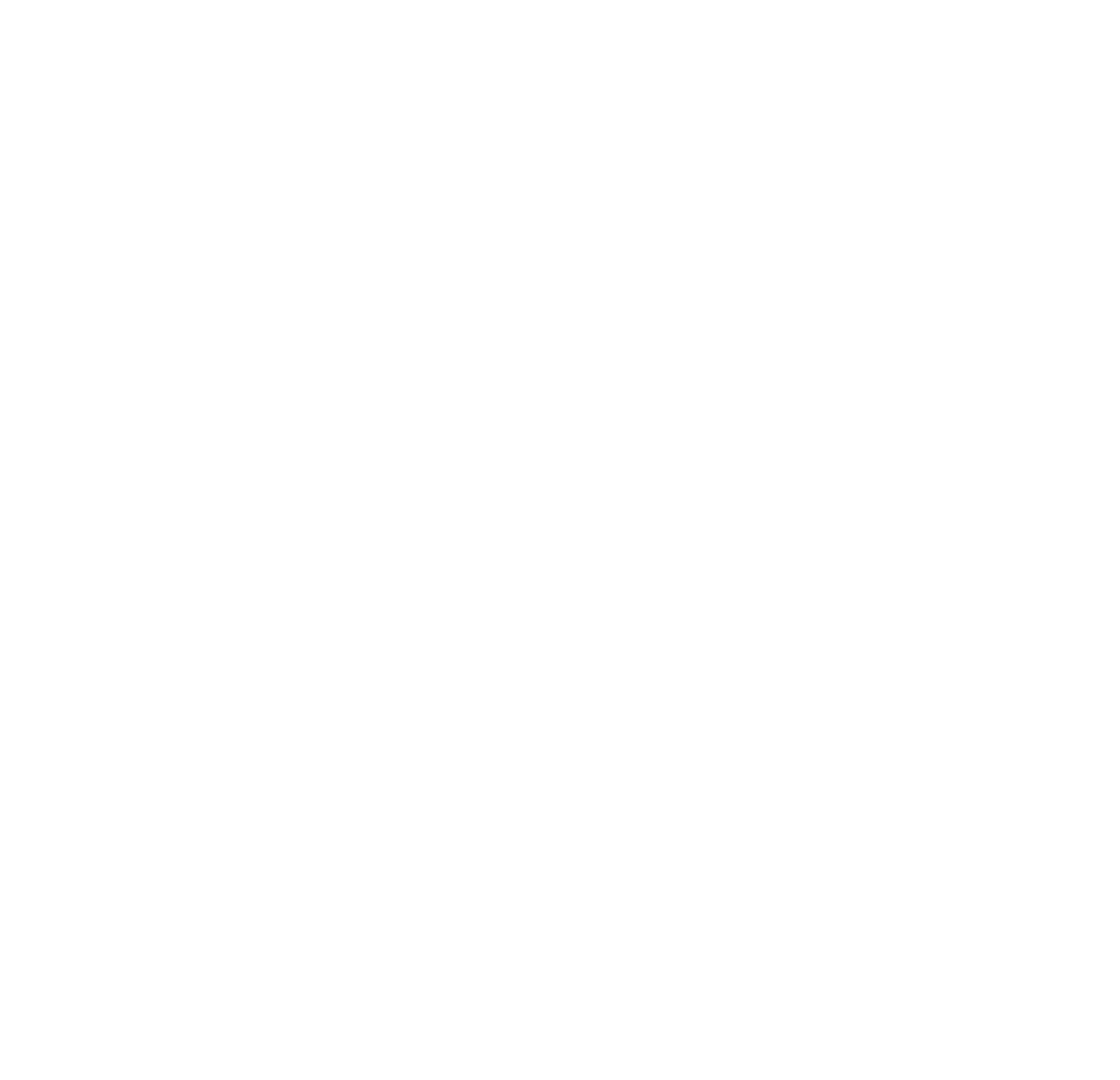

